\title{
On the Boundary of the Dispersion-Managed Soliton Existence
}

\author{
P. M. Lushnikov \\ Landau Institute for Theoretical Physics, Russian Academy of Sciences, ul. Kosygina 2, Moscow, 117334 Russia \\ Theoretical Division, MS-B284, Los Alamos National Laboratory, Los Alamos, New Mexico, 87545 \\ e-mail:lushnikov@lanl.gov
}

Received June 19, 2000; in final form, July 12, 2000

\begin{abstract}
A breathing soliton-like structure in dispersion-managed optical fiber system is studied. It is proved that, for negative average dispersion, the breathing soliton is forbidden, provided that the modulus of average dispersion exceeds a threshold which depends on the soliton amplitude. (O) 2000 MAIK "Nauka/Interperiodica".
\end{abstract}

PACS numbers: 05.45.Yv, 42.65.Tg, 42.79.Sz, 42.81.Dp

Propagation of optical pulse in nonlinear media with varying dispersion is both fundamental [1] and important applied problem [2-8] because the dispersionmanaged (DM) system, which is a system with periodic dispersion variation along an optical fiber, is one of the most prospective candidate for ultrafast high-bit-rate optical communication lines. Lossless propagation of optical pulse in DM fiber is described by a nonlinear Schrödinger equation (NLS) with periodically varying dispersion $d(z)$ :

$$
i u_{z}+d(z) u_{t t}+|u|^{2} u=0,
$$

where $u$ is the envelope of the optical pulse, $z$ is the propagation distance, and all quantities are dimensionless. Consider a two-step periodic dispersion map: $d(z)=d_{0}+\tilde{d}(z)$, where $\tilde{d}(z)=d_{1}$ for $0<z+n L<L_{1}$ and $\tilde{d}(z)=d_{2}$ for $L_{1}<z+n L<L_{1}+L_{2} ; d_{0}$ is the path-averaged dispersion; $d_{1}$ and $d_{2}$ are the amplitudes of dispersion variation subject to the condition $d_{1} L_{1}+d_{2} L_{2}=0$; $L \equiv L_{1}+L_{2}$ is the dispersion compensation period; and $n$ is the arbitrary integer number. Equation (1) also describes the pulse propagation in a fiber with losses compensated by periodically placed amplifiers, if the distance between amplifiers is much less than $L$.

In a linear regime, in which the nonlinear term in Eq. (1) is negligible, the periodical variation of dispersion is a way to overcome pulse broadening due to the chromatic dispersion, provided that the residual dispersion $d_{0}$ is small enough. However, in a real optical fiber, the nonlinear term in Eq. (1) is important because the optical pulse amplitude should be large enough to get high signal/noise ratio. One of the fascinating features of the DM system is the numerical observation of a space-breathing soliton-like structure, which is called the DM soliton, for both positive and negative residual dispersion $d_{0}$ [9]. This observation is in sharp contrast with the system described by the NLS with the constant dispersion, where the stable soliton propagation is pos- sible only for the positive dispersion [10], because the nonlinearity can continuously compensate the positive dispersion only. In the DM soliton, the balance between the nonlinearity and dispersion is achieved, on average, over the dispersion period $L$, which lifts the requirement of the positive dispersion sign. Nevertheless, it was never proved that the DM soliton really exists because there is a possibility that this is a rather longlived quasi-stable breathing pulse which decays on a long distance $z$. It is shown here that for negative $d_{0} \mathrm{DM}$ soliton can exist only if $\left|d_{0}\right|$ is small enough to allow nonlinear compensation of pulse broadening due to the dispersion over distance $L$.

Equation (1) can be written in the Hamiltonian form $i u_{z}=\delta H / \delta u^{*}$, where the Hamiltonian

$$
H=\int\left[d(z)\left|u_{t}\right|^{2}-\frac{|u|^{4}}{2}\right] d t
$$

is an integral of motion on each interval of a constant dispersion $d(z)=$ const. Equation (1) is reduced to usual NLS on such intervals. At points $z=n L$ and $z=n L+L_{1}$, where $n$ is an arbitrary integer number, the Hamiltonian experiences jumps due to jumps of the dispersion, although the value of $u$ is a continuous function of $z$ in these points. In contrast to the Hamiltonian, the timeaveraged optical power $N=\int|u|^{2} d t$ or number of particles in the quantum mechanical interpretation of NLS (in this interpretation, the coordinate $z$ means some "time" and actual time $t$ has a meaning of "coordinate") is an integral of motion for all $z$. Consider $z$ dependence of the quantity $A=\int t^{2}|u|^{2} d t . A / N$ is the average width of a time distribution of $u$, or simply $\left\langle t^{2}\right\rangle$ in the quantum mechanical interpretation of NLS. 
Using (1) and integrating by parts, one gets for the first $z$ derivative

$$
A_{z}=d(z) \int 2 i t\left(u u_{t}^{*}-u^{*} u_{t}\right) d t
$$

In a similar way after the second differentiation with respect to $z$, one gets

$$
A_{z z}=4 d H+4 d^{2} X+\frac{d z}{d} A_{z}
$$

where $X \equiv \int\left|u_{t}\right|^{2} d t$. It follows from Eq. (3), which is often called the virial theorem (see, e.g., $[11,12]$ ), that $A_{z}$ experiences finite jumps corresponding to jumps of a step-wise function $d(z)$ :

$$
\begin{aligned}
& \left.A_{z}\right|_{z=L_{1}+0}=\left.\frac{d_{0}+d_{2}}{d_{0}+d_{1}} A_{z}\right|_{z=L_{1}-0}, \\
& \left.A_{z}\right|_{z=L+0}=\left.\frac{d_{0}+d_{1}}{d_{0}+d_{2}} A_{z}\right|_{z=L-0} .
\end{aligned}
$$

Set $X(z)=X_{0}+\delta X(z), X(0) \equiv X_{0}$; then one can integrate Eq. (4) over intervals $\left(0, L_{1}\right),\left(L_{1}, L\right)$ :

$$
\begin{gathered}
\left.A_{z}\right|_{z=L_{1}-0}=\left.A_{z}\right|_{z=0+0} \\
+4 \int_{0}^{L_{1}}\left[\left(d_{0}+d_{1}\right) H_{1}+\left(d_{0}+d_{1}\right)^{2} X\right] d z, \\
\left.A_{z}\right|_{z=L-0}=\left.A_{z}\right|_{z=L_{1}+0} \\
+4 \int_{L_{1}}^{L}\left[\left(d_{0}+d_{2}\right) H_{2}+\left(d_{0}+d_{2}\right)^{2} X\right] d z,
\end{gathered}
$$

where

$$
\begin{gathered}
H_{1}=\left(d_{0}+d_{1}\right) X_{0}-Y_{0}, \\
H_{2}=\left(d_{0}+d_{2}\right) X_{0}-Y_{0}-\left.\left(d_{1}-d_{2}\right) \delta X\right|_{z=L_{1}}
\end{gathered}
$$

are the Hamiltonian values on intervals $\left(0, L_{1}\right),\left(L_{1}, L\right)$ respectively,

$$
Y(z) \equiv \int \frac{|u|^{4}}{2} d t, \quad Y_{0} \equiv Y(0) .
$$

Here, the conservation of $H_{1}$ on interval $\left(0, L_{1}\right)$ is used in deriving expression for $\mathrm{H}_{2}$.

The DM soliton solution of Eq. (1) (see [13]) is given by $u=\tilde{u}(z, t) \exp (i k z)$, where $k$ is an arbitrary real constant and $\tilde{u}(z+L, t)=\tilde{u}(z, t)$ is a periodic function of $z$, $\left.\tilde{u}(z, t)\right|_{|t| \rightarrow \infty} \longrightarrow 0$. Thus, for DM soliton $\left.A_{z}\right|_{z=L+0}=$
$\left.A_{z}\right|_{z=0+0}$. This condition can be recast, via Eqs. (5)-(7), into the form:

$$
\begin{aligned}
& L\left(d_{1}+d_{0}\right)\left[2 d_{0} X_{0}-Y_{0}+\left.\left(d_{1}-d_{2}\right) \frac{L_{2}}{L} \delta X\right|_{z=L_{1}}\right] \\
& +\int_{0}^{L_{1}}\left(d_{0}+d_{1}\right)^{2} \delta X d z+\int_{L_{1}}^{L}\left(d_{0}+d_{2}\right)^{2} \delta X d z=0 .
\end{aligned}
$$

The next step is to consider $\delta X(z)$ dependence. Using Eq. (1) and integrating by parts, one can get

$$
X_{z}=4 \int \phi_{t} R_{t} R^{3} d t
$$

where $u \equiv R e^{i \phi}, \phi$ and $R$ are real, $R \geq 0$. Consider an upper bound of $X_{z}$, which is given by a chain of inequalities:

$4 \int \phi_{t} R_{t} R^{3} d t \leq 4 \max _{t}\left(R^{2}\right) \int\left|\phi_{t} R_{t} R\right| d t \leq 4 X^{3 / 2} N^{1 / 2}$,

where the following inequalities are used:

$$
\begin{gathered}
2 \phi_{t} R_{t} R \leq\left(\phi_{t} R\right)^{2}+R_{t}^{2}, \\
\max _{t}\left(R^{2}\right) \leq \int_{-\infty}^{t}\left|\left(R^{2}\right)_{t^{\prime}}\right| d t^{\prime} \\
\leq \int\left|\left(R^{2}\right)_{t}\right| d t \leq 2 \int R\left|R_{t}\right| d t \leq 2 N^{1 / 2} X^{1 / 2}
\end{gathered}
$$

(in the last expression, the Cauchy-Schwarz inequality is also used). Equations (9) and (10) can be integrated over $\mathrm{z}$ to give (it is assumed below that $\left.2 X_{0}^{1 / 2} N^{1 / 2} \max \left(L_{1}, L_{2}\right)<1\right)$ :

$$
X \leq X_{0} /\left(1-2 X_{0}^{1 / 2} N^{1 / 2} z\right)^{2}
$$

In a similar way, using inequality $X_{z} \geq-4 \int\left|\phi_{t} R_{t}\right| R^{3} d t$ following from Eq. (9), one can get the lower bound for $X(z)$ :

$$
X \geq X_{0} /\left(1+2 X_{0}^{1 / 2} N^{1 / 2} z\right)^{2} .
$$

For the DM soliton $X(L)=X_{0}$, and, thus, it is more convenient to use for $L_{1}<z<L$ similar inequalities:

$$
\begin{gathered}
\frac{X_{0}}{\left(1+2 X_{0}^{1 / 2} N^{1 / 2}(L-z)\right)^{2}} \\
\leq X \leq \frac{X_{0}}{\left(1-2 X_{0}^{1 / 2} N^{1 / 2}(L-z)\right)^{2}} .
\end{gathered}
$$


Equations (8), (12)-(14) result in inequality:

$$
\begin{gathered}
\left|2 d_{0} X_{0}-Y_{0}\right| \leq \frac{\left|d_{1}-d_{2}\right| L_{2} X_{0}}{L}\left[\frac{1}{\left(1-2 X_{0}^{1 / 2} N^{1 / 2} L_{1}\right)^{2}}-1\right] \\
+\frac{2 X_{0}^{3 / 2} N^{1 / 2}}{\left|d_{0}+d_{1}\right| L}\left[\frac{\left(d_{0}+d_{1}\right)^{2} L_{1}^{2}}{1-2 X_{0}^{1 / 2} N^{1 / 2} L_{1}}+\frac{\left(d_{0}+d_{2}\right)^{2} L_{2}^{2}}{1-2 X_{0}^{1 / 2} N^{1 / 2} L_{2}}\right] .
\end{gathered}
$$

Equation (15) is the main result of this paper. Equation (15) is a consequence of initial assumption that the DM soliton exists for given parameters $L_{1}, L_{2}, d_{0}, d_{1}, d_{2}$ and integral values $X_{0}, Y_{0}, N$, which depend on $\left.u\right|_{z=0}$ only. Thus, the DM soliton can exist only if this inequality is fulfilled.

Note that if one assumes uniqueness of DM soliton solution for given $k$ and soliton width, then, as shown in [13], $|u|_{z=0}=|u|_{z=L_{1}}$. In such a case the term $\left.\delta X\right|_{z=L_{1}}$ in Eq. (8) vanishes and instead of (15) one can get a more strict inequality. However this possibility is disregarded here for the sake of generality.

To clarify physical consequences of Eq. (15), consider an optical pulse with a typical amplitude $p$ and a typical time width $t_{0}$. Then, $N \sim|p|^{2} t_{0}, X_{0} \sim|p|^{2} / t_{0}$ and, thus, $X_{0}^{1 / 2} N^{1 / 2} L \sim L / Z_{n l}$, where $Z_{n l}=1 /|p|^{2}$ is the characteristic nonlinear length. In typical experimental conditions, the nonlinearity is small: $L / Z_{n l} \ll 1$ and denominators in (15) can be expanded in series to give

$$
\begin{gathered}
\left|2 d_{0} X_{0}-Y_{0}\right| \leq \frac{2 X_{0}^{3 / 2} N^{1 / 2}}{L} \\
\times\left[2\left|d_{1}-d_{2}\right| L_{1} L_{2}+\left|d_{0}+d_{1}\right| L_{1}^{2}+\frac{\left(d_{0}+d_{2}\right)^{2} L_{2}^{2}}{\left|d_{0}+d_{1}\right|}\right] \\
+O\left(\frac{d_{1} L^{3}}{t_{0} Z_{n l}^{3}}\right) .
\end{gathered}
$$

Provided that $d_{0}$ is negative, both terms on the left-hand side of Eq. (16) have the same sign and, thus, the righthand side should be greater or equal to $2\left|d_{0}\right| X_{0}+Y_{0}$. Assuming $d_{1} \gg\left|d_{0}\right|$, one can get from Eq. (16) the following estimate $\left(Y_{0} \sim t_{0} / Z_{n l}^{2}\right)$ :

$$
\frac{2\left|d_{0}\right|}{t_{0} Z_{n l}}+\frac{t_{0}}{Z_{n l}^{2}} \lessgtr \frac{4 L_{1} d_{1}}{Z_{n l}^{2} t_{0}}\left(1+\frac{L_{1}}{L}\right) .
$$

Consider the strong dispersion management limit $Z_{\text {disp }} / L \ll 1$, where $Z_{\text {disp }} \equiv t_{0}{ }^{2} / d_{1}$ is the typical dispersion length. This limit implies that the optical pulse experiences strong oscillation at each period $L$ due to the dispersion. Then Eq. (17) reduces to

$$
-\frac{d_{0}}{d_{1}} \leqslant \frac{6 L_{1}}{Z_{n l}}\left(1+\frac{L_{1}}{L}\right)
$$

i.e., a nonlinearity (amplitude of the optical pulse) should be strong enough to allow the DM soliton solution to exist for a given negative $d_{0}$.

Equation (15) gives the necessary, but not sufficient, condition for the existence of the DM soliton. In other words, the violation of inequality (15) means that the DM soliton is forbidden. Of course, it would be interesting to find to what extent this necessary condition for existence is close to the sufficient one. In general, this could be done only if one found the DM soliton analytically.

Here, one can only mention that there is a qualitative correspondence between the threshold of DM soliton existence, following from the analytical condition (15) and from the numerical investigation of the DM soliton. Namely, the maximum value of $\left|d_{0}\right|\left(d_{0}<0\right)$ for which the DM soliton exists grows with increase in the dispersion map strength $L / Z_{\text {disp }}$, according to both numerics (see, e.g., $[14,15]$ ) and analytical condition (16). It also follows from Eq. (18) that for asymmetric dispersion map $L_{1} \neq L_{2}$ the maximum possible value of $\left|d_{0}\right|$ grows as $L_{1}$ increases (for fixed $L, Z_{n l}, d_{1}$ ), in correspondence with Fig. 3 in [15].

Equation (15) has also a clear physical meaning in another limit $d_{0} / d_{1} \gg L / Z_{n l}, Z_{d i s p} \gg L$, and $Z_{n l} \gg L$, in which Eq. (15) reduces to

$$
\left(2 d_{0} X_{0}-Y_{0}\right) / Y_{0}=O\left(L / Z_{\text {disp }}\right) \ll 1 .
$$

Equality $2 d_{0} X_{0}=Y_{0}$ exactly corresponds to one-soliton solution of the NLS with dispersion $d_{0}$ (see [10]), where the dispersion $d_{0}$ and the nonlinearity continuously balance each other. Thus, in the limit $Z_{\text {disp }} \gg L$, which is called a weak dispersion limit, we recover usual NLS describing the path-averaged (over space period $L$ ) DM soliton dynamics, provided $d_{0}$ is large enough. A weak dispersion management limit was studied earlier [1, 16-18]. Note that an additional condition $d_{0} / d_{1} \gg$ $L / Z_{n l}$ allows the amplitude $d_{1}$ of the dispersion variation to be still much higher than $d_{0}$ because one assumes $L \ll Z_{n l}$.

To summarize the necessary analytical condition (15) for the existence of the DM soliton is established. From the physical point of view, this condition means that the DM soliton solution can exist only if the nonlinearity is strong enough to compensate the pulse broadening due to the negative value of the average dispersion $d_{0}$. Note that estimates in Eqs. (16)-(19) are only given here for a physical interpretation of the analytical condition (15). So far, the DM soliton solution was obtained numerically $[3,4,14]$ and by the variational [5] and other perturbative approaches [19-21]. These results are in agreement with the condition (15). But analytical proof of the existence of the DM soliton in the parameter region satisfying condition (15), i.e. the sufficient condition for existence, is still an open question. 

sions.

The author thanks I.R. Gabitov for helpful discus-

The support was provided by the US Department of Energy, under contract W-7405-ENG-36, RFBR and the program of the Russian Government Support for Leading Scientific Schools.

\section{REFERENCES}

1. V. E. Zakharov, in Optical Solitons: Theoretical Challenges and Industrial Perspectives, Ed. by V. E. Zakharov and S. Wabnitz (Springer-Verlag, Berlin, 1999), p. 73; V. E. Zakharov and S. V. Manakov, Pis'ma Zh. Eksp. Teor. Fiz. 70, 573 (1999) [JETP Lett. 70, 578 (1999)].

2. C. Lin, H. Kogelnik, and L. G. Cohen, Opt. Lett. 5, 476 (1980).

3. M. Nakazawa and H. Kubota, Electron. Lett. 31, 216 (1995).

4. N. J. Smith, F. M. Knox, N. J. Doran, et al., Electron. Lett. 32, 54 (1996).

5. I. Gabitov and S. K. Turitsyn, Opt. Lett. 21, 327 (1996); Pis'ma Zh. Éksp. Teor. Fiz. 63, 814 (1996) [JETP Lett. 63, 861 (1996)].

6. S. Kumar and A. Hasegawa, Opt. Lett. 22, 372 (1997).

7. P. V. Mamyshev and N. A. Mamysheva, Opt. Lett. 24, 1454 (1999).

8. L. F. Mollenauer, P. V. Mamyshev, J. Gripp, et al., Opt. Lett. 25, 704 (2000).

9. J. H. B. Nijhof, N. J. Doran, W. Forysiak, and F. M. Knox, Electron. Lett. 33, 1726 (1997).

10. V. E. Zakharov and A. B. Shabat, Zh. Éksp. Teor. Fiz. 61, 118 (1971) [Sov. Phys. JETP 34, 62 (1972)].

11. V. E. Zakharov, Zh. Éksp. Teor. Fiz. 62, 1745 (1972) [Sov. Phys. JETP 35, 908 (1972)].

12. P. M. Lushnikov, Pis'ma Zh. Éksp. Teor. Fiz. 62, 447 (1995) [JETP Lett. 62, 461 (1995)].

13. S. K. Turitsyn, J. H. B. Nijhof, V. K. Mezentsev, and N. J. Doran, Opt. Lett. 24, 1871 (1999).

14. A. Berntson, N. J. Doran, W. Forysiak, and J. H. B. Nijhof, Opt. Lett. 23, 900 (1998).

15. A. Berntson, D. Anderson, N. J. Doran, et al., Electron. Lett. 34, 2054 (1998).

16. A. Hasegawa and Y. Kodama, Solitons in Optical Communications (Oxford Univ. Press, New York, 1995).

17. Yu. L. Lvov and I. R. Gabitov, chao-dyn/9907007 (1999).

18. S. B. Medvedev and S. K. Turitsyn, Pis'ma Zh. Éksp. Teor. Fiz. 69, 465 (1999) [JETP Lett. 69, 499 (1999)].

19. S. K. Turitsyn and V. K. Mezentsev, Pis'ma Zh. Éksp. Teor. Fiz. 67, 616 (1998) [JETP Lett. 67, 640 (1998)]; S. K. Turitsyn, Phys. Rev. E 58, 1256 (1998).

20. T. Lakoba and D. J. Kaup, Electron. Lett. 34, 1124 (1998).

21. P. M. Lushnikov, Opt. Lett. 25, 1144 (2000). 